\title{
Surgical Treatment of Loffler Endocarditis in an 8-Year-Old Girl
}

\author{
Zhangke Guo, MD, Fan Fan, MD, Pei Li, MD, Xiaofeng Li, MD, Ning Ma, MD Libing Fu, MD, \\ Song Bai, MD, Feng Tong, MD, Jia Zheng, MD, Qilin Li, MD \\ Pediatric Cardiac Center, Beijing Children's Hospital \& Capital Medical University, Beijing, China
}

\section{ABSTRACT}

Background: Idiopathic hyper eosinophilic syndrome (HES) is a rare disease characterized by a sustained increase in eosinophilia. Heart involvement is called Loffler endocarditis. Loffler endocarditis is a serious complication of hyper eosinophilia syndrome, which is characterized by a special type of fibrotic endocarditis. Loffler endocarditis is an inflammatory cardiac condition characterized by eosinophilic infiltration in the heart. The overall prognosis for patients with Loffler endocarditis is very poor.

Methods: In this article we report an 8-year-old girl who was diagnosed as having Loffler endocarditis in thrombotic phase and was successfully treated with surgery.

Results: Our patient had a good prognosis during the half-year follow-up. She had no symptoms of heart failure and echocardiography findings were normal.

Conclusion: The cardiac damage occurred in a threestage process: the necrotic, thrombotic, and fibrotic stages. This unusual but sometimes life-threatening disease is often detected in the late phase, resulting in no curative strategy available to reverse the disease process. The overall prognosis of patients with Loffler endocarditis is very poor. Current treatments include anticoagulation and anti-eosinophils therapy, and surgery only in selected cases. Surgical treatment of HES in adolescents is very rare. The present case illustrates that with well-controlled peripheral eosinophilia, proper surgical treatment in selected patients can improve their prognosis in the near future but long-term follow-up is necessary.

\section{INTRODUCTION}

Idiopathic hyper eosinophilic syndrome (HES) is a rare disease characterized by sustained increase in eosinophilia. Lesions can affect various organs and systems. The global prevalence rate is unknown, and the prognosis is poor. At present, most of the literature reports are based on cases. Heart involvement may be present in $50 \%$ to $60 \%$ of patients, and is called Loffler endocarditis [Niemeijer 2012]. Loffler endocarditis is a serious complication of

Received September 19, 2020; received in revised form October 19,2020; accepted November 2, 2020.

Correspondence: Dr. Xiaofeng Li, Pediatric Cardiac Center, Beijing Children's Hospital \& Capital Medical University, Beijing, China; (e-mail: xiaofengl2000@sina.com). hyper eosinophilia syndrome, which is characterized by a special type of fibrotic endocarditis. Loffler endocarditis is an inflammatory cardiac condition characterized by eosinophilic infiltration in the heart. The cardiac damage occurs in three stage processes: the necrotic, thrombotic, and fibrotic stages. This unusual but sometimes life-threatening disease is often detected in the late phase, resulting in no curative strategy available to reverse the disease process. The overall prognosis of patients with Loffler endocarditis is very poor. Current treatments include anticoagulation and antieosinophils therapy, and surgery in only selected cases. Surgical treatment of HES in adolescents is very rare. In this article we report an 8-year-old girl who was diagnosed as having Loffler endocarditis in the thrombotic phase and who was successfully treated with surgery.

\section{CASE REPORT}

An 8-year-old girl was admitted to our hospital in December 2019 complaining of a rash for 11 days, abdominal pain for 8 days, and fever and cough for 3 days. On physical examination, we found symmetrical distribution of dense reddish skin rash on limbs, ears, and buttocks, no fading of pressure, slightly higher than skin, partially fused into pieces. Laboratory studies showed a white blood cell count of 14.83 $\times 10^{9} / \mathrm{L}$ with $41.9 \%$ eosinophils and an absolute value of 6.21

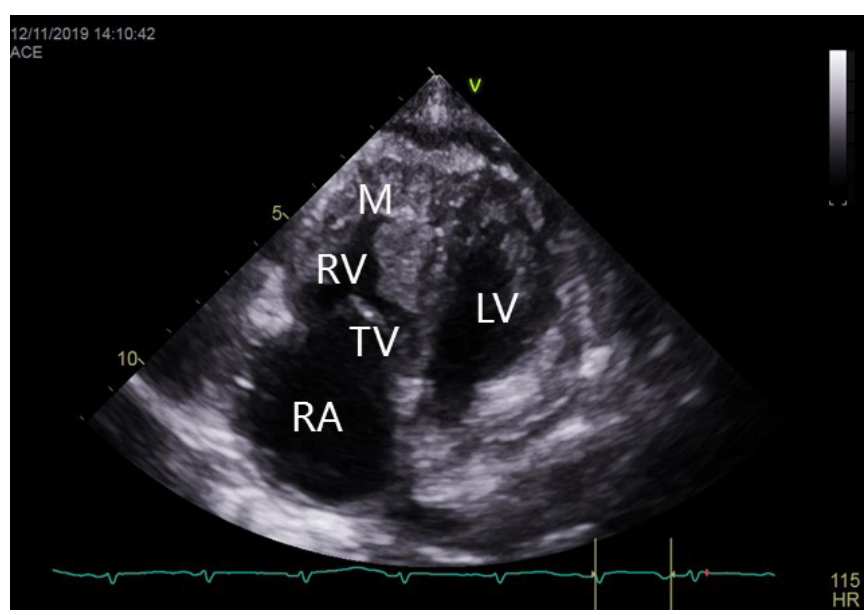

Figure 1. Transthoracic echocardiogram shows a large mass in the right ventricle and the right atrium enlarged. RA indicates right atrium; TV, tricuspid valve; LV, left ventricle; LA, left atrium; $M$, mass. 


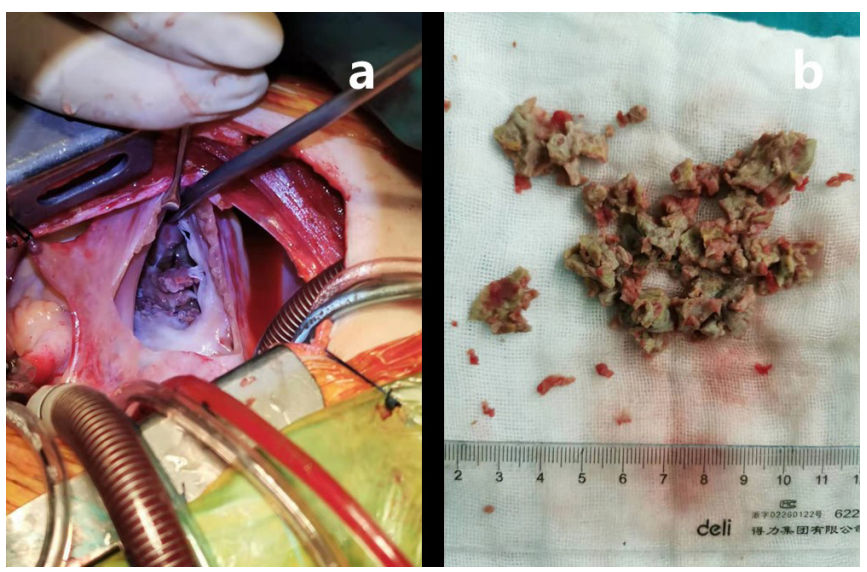

Figure 2. (a) Transatrial incision shows the thrombus tissue filling the right ventricle. (b) Surgical specimens show a lot of thrombus tissue.

$\times 10^{9} / \mathrm{L}$ (normal values, $0.5-5 \%$ or $0.05-3 \times 10^{9} / \mathrm{L}$ ). A bone marrow biopsy showed marked eosinophilia with $42.5 \%$ but no evidence of abnormal myeloid maturation. Liver and kidney function,troponin-I level, and creatinine kinase level were normal. Rheumatoid factor erythrocyte sedimentation rate, serum immunoglobulins, and antinuclear antibodies were all within normal limits. The stool, urine, and parasite analysis were normal. The chest $\mathrm{X}$-ray suggested pneumonia, and the abdominal ultrasound examination and transthoracic echocardiography were normal. We used linezolid (10 $\mathrm{mg} / \mathrm{kg}$, q $8 \mathrm{~h}$, oral), combined with Minocycline (2 mg/kg, q $12 \mathrm{~h}$, oral). After 13 days of anti-infective and anti-eosinophils therapy, the white blood cell count was $16.83 \times 10^{9} / \mathrm{L}$ with $13.9 \%$ eosinophils and an absolute value of $1.58 \times 10 \% / \mathrm{L}$. The patient still had intermittent fever with a higher temperature of up to 38.5 degrees. Facial edema, anorexia, and swelling of the liver were evident. The chest X-ray suggested aggravated pneumonia and pleural effusion. Transthoracic echocardiography revealed a large mass which nearly obliterated the right ventricular cavity and extended into the right ventricular outflow tract. The right atrium was enlarged, moderate tricuspid regurgitation was noted, and moderate pericardial effusion was observed (Figure 1). We give methylprednisolone upon noticing the mass in the RV before the operation. (25 mg, q $12 \mathrm{~h}$, intravenous infusion). We performed emergency surgery for this patient. We operated via median sternotomy and under cardiopulmonary bypass. We found a large thrombus involving almost the entire right ventricle (Figure 2). The tricuspid was severely surrounded by the thrombus tissue; thrombectomy and tricuspid valvuloplasty were performed. The duration of extracorporeal circulation was 107 minutes and the aortic blockage duration was 82 minutes. The patient was extubated 10 hours after the operation in the Cardiac Care Unit (CCU) and no further complications were reported. We used antibiotics to prevent infection, using furosemide and spironolactone to reduce cardiac load, and Nadroparin 0.1 $\mathrm{mL} / 10 \mathrm{~kg} \mathrm{q} 12 \mathrm{~h}$ subcutaneous injection to prevent thrombus recurrence. The goal is to maintain APTT at 1.5 times the normal value.

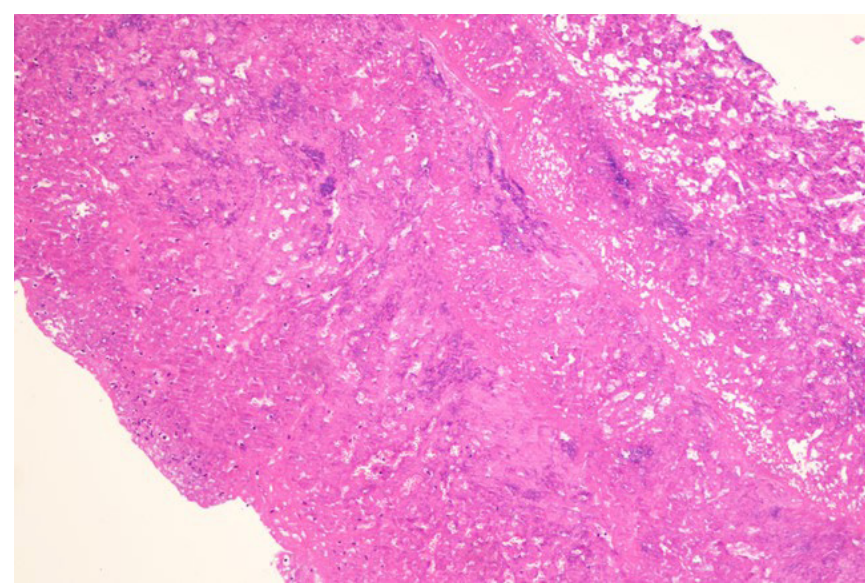

Figure 3. Pathological tissue are pink, with degeneration, no structure, local rich in mucus with scattered and focal neutrophils, with a small number of small lymphocytes, eosinophils (HE staining, original magnification $\times 10$ ).

Histologic specimen showed thrombus; all of the pathological tissue was pink, with degeneration, no structure, local rich in mucus with scattered and focal neutrophils, with a small number of small lymphocytes, eosinophils (HE staining, original magnification $\times 10)$ (Figure 3$)$.

\section{DIscussion}

There are obvious individual differences in the clinical manifestations of HES, ranging from asymptomatic, multiple organ involvement to death. This pathology may involve many organs and systems, such as the skin, lungs, nervous system, gastrointestinal tract, kidneys, and heart. Therefore, diagnosis may be a challenge. The main morbidity and mortality of HES patients are cardiovascular complications [Flanigan 2017]. Heart involvement may be present in $50 \%$ to $60 \%$ of patients, which constitutes Loffler endocarditis.

Due to infiltration and degranulation of eosinophils, toxic proteins are released, causing tissue inflammation and late fibrosis resulting in secondary damage to the myocardium and endocardium. This is usually characterized by thickening of the endocardium and dilation of the atrium. Doppler echocardiography can show hyperechoic mass in the heart cavity, suggesting fibrosis or thrombosis, and is often located in the apical area of the left and right ventricles [Sawayama 2015; Nakashima 2014].

Echocardiography and imaging studies may be the main tools for assessing cardiac anatomy and function. However, Loffler endocarditis requires pathological diagnosis, so endocardial biopsy remains the gold standard. In some cases, myocardial biopsy may be a dangerous operation. Therefore, clinicians should personally evaluate each individual before performing such invasive procedures. In addition, when evaluating the possibility of Loffler endocarditis, it is important to exclude the main differential diagnosis of HES, including hyper eosinophils secondary to hypersensitivity and 
parasitic infections. In this case, preoperative laboratory studies showed peripheral hyper eosinophilia and echocardiography showed typical intraventricular space-occupying changes. The diagnosis of Loffler endocarditis was basically established, and postoperative pathological specimens confirmed the previous diagnosis. The pathological features of Loffler endocarditis include thickening of endocardial fibers, leading to a decrease in cardiac volume, thrombosis, and restrictive cardiomyopathy. The clinical manifestations are heart failure, thromboembolic events, and atrial fibrillation.

The main purpose of treatment is to reduce the level of eosinophils in peripheral blood and tissues, prevent terminal organ damage, and avoid the occurrence of adverse thrombotic events. Heart failure in Loffler endocarditis is mainly due to limited diastolic function of the heart. Treatment includes intravenous diuretics to reduce the preload of the heart. In addition, for symptomatic patients, the first-line drugs of choice are corticosteroids, followed by cytotoxic drugs such as hydroxyurea and immunomodulators such as alpha-interferon. Glucocorticoid therapy is clinically proven to be effective for HES, which can improve the condition of myocardial injury and normalize peripheral hyper eosinophilia [Yamamoto 2018; Zhuang 2015].

Secondary treatment should target cardiac complications such as heart failure and the presence of intracardiac thrombosis. Sometimes, cardiovascular complications require surgical treatment. Surgical experience is limited to cases where the heart valve function of patients due to HES is affected, but in more serious cases, acute surgical intervention may be necessary.

In this case reported by our center, due to the large amount of thrombus tissue filling the right ventricle in a short time, the volume of the right ventricle was significantly reduced, the tricuspid valve was wrapped in the thrombus tissue, the opening of tricuspid valve was restricted, and there was a risk of pulmonary embolism caused by thrombosis. Therefore, the heart thrombus was removed under emergency cardiopulmonary bypass surgery.

Our patient had a good prognosis during the half-year follow-up. She had no symptoms of heart failure and echocardiography findings were normal. This result might be due to the proper operation as well as the control of peripheral eosinophilia before and after the surgical procedure. Loffler endocarditis caused by hyper eosinophilia syndrome is unusual and fatal in children. The present case illustrates that with well-controlled peripheral eosinophilia, proper surgical treatment in selected patients can improve their prognosis in the near future, but long-term follow-up is necessary.

\section{REFERENCES}

Flanigan MJ, Velez MR, Ruden EA, Lilly SM. 2017. Löffler endocarditis and endomyocardial fibrosis. J Am Osteopath Assoc 117:601.

Nakashima T, Funada A, Kanzaki H, et al. 2014. Massive mobile thrombus in the left ventricle due to Löffler endocarditis complicated with dilated cardiomyopathy. Circ J 78:1992-4.

Niemeijer ND, van Daele PL, Caliskan K, Oei FB, Loosveld OJ, van der Meer NJ. 2012. Löffler endocarditis: a rare cause of acute cardiac failure. J Cardiothorac Surg 7:109.

Sawayama Y, Itoh H, Sakai H, Horie M. 2015. Löffler endocarditis and lacking heart. Intern Med 54:3093.

Yamamoto M, Seo Y, Ishizu T, Aonuma K. 2018. Reversible aortic valve stenosis with Löffler endocarditis. Eur Heart J 39:2332.

Zhuang Q, Zheng ZY, Mao W, et al. 2015. Right ventricular apical obstruction in a patient with hypereosinophilia: Löfller endocarditis. Heart Lung 44:165-9. 